\title{
Extending the Reach of GPS-assisted Femtocell Synchronization and Localization Through Tightly-Coupled Opportunistic Navigation
}

\author{
Kenneth M. Pesyna Jr., Kyle D. Wesson, and Robert W. Heath Jr. \\ Department of Electrical and \\ Computer Engineering \\ The University of Texas at Austin \\ Email: \{kpesyna, kyle.wesson, rheath\}@mail.utexas.edu
}

\author{
Todd E. Humphreys \\ Department of Aerospace Engineering and \\ Engineering Mechanics \\ The University of Texas at Austin \\ Email: todd.humphreys@mail.utexas.edu
}

\begin{abstract}
A strategy known as Tightly-Coupled Opportunistic Navigation (TCON) is proposed for extending the penetration of GPS-assisted femtocells in weak-signal indoor environments. Standards and regulatory agencies impose strict time, frequency, and location requirements on femtocells. These can be met with GPS aiding to unparalleled time and positioning accuracies, but GPS signals are extremely weak and thus difficult to acquire indoors. The results of this paper suggest that a TCON solution fusing GPS with CDMA cellular signals offers significant sensitivity gains over state-of-the-art assisted-GPS receivers, allowing GPS-synchronized femtocells to be deployed in $90 \%$ of all residences.
\end{abstract}

\section{INTRODUCTION}

Femtocells are low-power base stations operated by cellular providers typically installed inside homes, businesses, and in weak-signal environments [1]. Cellular air interface standards impose strict time and frequency requirements on femtocell synchronization [2]. There exist today a number of synchronization solutions which claim to meet some or all of these requirements, including Cellular Network Listen [3], solutions based on the Precision Time Protocol (PTP/IEEE 1588) [4], and solutions based on the Global Positioning System (GPS) [5], [6].

In addition to synchronization requirements, designers of femtocells must consider location requirements. Regulations on femtocell localization are not currently well defined. Phase I of the U.S. Federal Communications Commission (FCC) E911 mandate states that a carrier must be able to provide "the location of the base station or cell site receiving the 911 call" [7]. The FCC has not yet specified what this requirement entails for femtocells. However, a recent FCC request for comments [8] suggests that, quite understandably, the FCC would like femtocell location accuracy to be roughly equivalent to macrocell location accuracy, or within about 10 meters. If such a strict location requirement is eventually adopted, then the location by-product of the Cellular Network Listen and PTP synchronization technologies would prove inadequate, as these struggle to deliver location accuracies under 100 meters [9], [10]. In contrast, GPS provides sub-10 meter location accuracy [11] in addition to excellent timing and frequency synchronization [5], [6]. Thus, despite the difficulty of exploiting its signals indoors, the GPS's global, freely-available signals and promise of accurate timing and positioning make GPS a hard habit to break.

State-of-the-art GPS technology has enabled receivers using assisted GPS, or A-GPS, to acquire and track GPS signals with a carrier-to-noise ratio, $C / N_{0}$, as low as $14 \mathrm{~dB}-\mathrm{Hz}$ [12]. Unfortunately, this is not good enough to enable broad indoor femtocell deployment. Indoor attenuation models and empirical studies indicate that a significant percentage of deployed femtocells would see GPS signals below $C / N_{0}=14$ $\mathrm{dB}-\mathrm{Hz}$ [13], [14]. A realistic goal for indoor GPS might be to acquire and track signals with $C / N_{0}$ as low as $5 \mathrm{~dB}-\mathrm{Hz}$, approximately a 9-dB improvement over the state-of-the-art. Such sensitivity would allow GPS-synchronized femtocells to be deployed in $90 \%$ of residences [13].

This paper proposes a new technique called Tightly-Coupled Opportunistic Navigation (TCON) to close this 9-dB sensitivity gap in GPS-assisted femtocells. TCON exploits non-GPS "signals of opportunity" (SOPs) to assist and enhance conventional GPS navigation techniques in weak signal environments [15]. Compared with the most advanced incarnations of AGPS [16], [17], TCON is superior because it fuses GPS and SOP observables at the carrier phase level instead of at the so-called group delay level. The resulting increased aiding precision reduces uncertainty in the GPS acquisition search and enables extended coherent integration, both of which lead to dramatic improvements in GPS sensitivity.

\section{Femtocell Time, Frequency, and Location REQUIREMENTS}

\section{A. Time and Frequency Requirements}

Femtocells are required to meet minimum time and frequency synchronization requirements imposed by cellular standards. Time synchronization requirements are necessary to avoid interference and negotiate seamless handovers in synchronized networks, particularly in time-division duplex (TDD) networks. Frequency synchronization requirements are necessary to maintain frequency alignment with the macrocells 
TABLE I

TIME AND FREQUENCY SYNCHRONIZATION REQUIREMENTS [2]

\begin{tabular}{|l|l|l|}
\hline Standard & Time & Frequency \\
\hline CDMA2000 & $10 \mu \mathrm{s}$ & $100 \mathrm{ppb}$ \\
\hline GSM & N/A & $100 \mathrm{ppb}$ \\
\hline WiMAX & $1 \mu \mathrm{s}$ (TDD) & $8 \mathrm{ppm}$ \\
\hline LTE & $3 \mu \mathrm{s}$ (TDD) & $250 \mathrm{ppb}$ \\
\hline WCDMA & $2.5 \mu \mathrm{s}$ (TDD) & $250 \mathrm{ppb}$ \\
\hline TD-SCDMA & $2.5 \mu \mathrm{s}$ & $100 \mathrm{ppb}$ \\
\hline
\end{tabular}

to avoid spectrum interference, particularly in frequencydivision duplex (FDD) networks.

Table I outlines time and frequency synchronization requirements imposed by various $3 \mathrm{G}$ and $4 \mathrm{G}$ cellular standards. The WiMAX, LTE, WCDMA, and TD-SCDMA standards are particularly strict in their time requirements whereas the CDMA2000, GSM and TD-SCDMA standards are particularly strict in their frequency requirements.

\section{B. Location Requirements}

Femtocells must also adhere to location requirements set forth by regulatory agencies and femtocell operators. Femtocell location requirements are motivated by three primary concerns. The first is spectrum use. Because femtocell operators are often licensed spectrum by federal regulatory agencies, they must ensure that femtocell users do not transport and activate devices in areas that the operator does not have a license to transmit [18]. The second concern is operator control. If a user takes a femtocell out of its licensed area or out of the country, operators may wish to impose additional charges on that user or even disable the femtocell to avoid losing revenue from roaming fees. The third concern, and the one that leads to the most stringent requirement, is emergency caller location identification. In the United States, the Federal Communications Commission (FCC) has passed the Enhanced 911 or E911 legislation. Phase I of this legislation mandates the identification of the location of the cellular base station [7]. Some carriers take a strict interpretation of this regulation and assume that it encompasses femtocells. Furthermore, it is most-likely assumed by these carriers that the location accuracy of femtocells must be on the same order as the accuracy achieved by macrocell base stations. Phase II of the legislation mandates that carriers must determine the caller's coordinates and be accurate to within 50 meters for 67 percent of emergency calls and 150 meters for 95 percent of calls. Assuming a femtocell radius of 25 meters, a femtocell's broadcast range is small enough such that its location is an adequate proxy for that of the mobile equipment it serves. Thus for most femtocells, if a strict interpretation of Phase I of the FCC mandate is met, so is Phase II.

\section{Femtocell SynchronizATION AND LOCALIZATION SOLUTIONS}

There are a number of solutions being investigated as a viable means to provide femtocells with location and timing information. Three of the most promising technologies are (1) Cellular Network Listen, (2) the Precision Time Protocol, and (3) the Global Positioning System.

\section{A. Cellular Network Listen}

Cellular Network Listen makes use of signals from macrocell base stations for femtocell synchronization. These signals can offer timing accuracy as low as a few microseconds and frequency accuracy within $50 \mathrm{ppb}$ [10]. However, cellular network location accuracy has been shown to be anywhere from 50-300 meters [9]. This would not satisfy a strict interpretation of the E911 mandate.

\section{B. The PTP-Based Solution}

The Precision Time Protocol (PTP) as defined in the IEEE 1588 standard would be ideal for femtocell time and frequency synchronization since an operator could take advantage of the user's readily available network backhaul for this synchronization. PTP works under a master-slave clock architecture: the master clock provides a timing and frequency reference to the local clock through a series of network segments and boundary clocks [4].

Despite the attractiveness of PTP's universal availability, the protocol suffers from a few drawbacks. First, the PTP algorithm assumes symmetry in the uplink and downlink packet delay times in order to calculate and correct the local clock timing error. Unfortunately, asymmetry is often present on residential internet connections where bandwidth is heavily shared and packet congestion can occur. This asymmetry coupled with variations in packet delay caused by network jitter degrades the accuracy of femtocell timing synchronization. Typical accuracy is around 5-20 ms [10]. Second, the location determination of the femtocell using the PTP approach is limited to the location derived from the IP address associated with the network connection. These location estimates are often very coarse (city-level) [19]; furthermore, femtocell operators may not have access to this information.

\section{The GPS-Based Solution}

The Global Positioning System (GPS) was initially developed in 1973 to enable accurate navigation for the military. Since May 2000, when the intentional degrading of the GPS signal known as Selective Availability was permanently turned off, GPS receivers have become much more accurate and pervasive. Not only are they being used as personal navigation devices, but they are being exploited for their time and frequency synchronization capabilities as well. In fact, much of the United States' critical infrastructure relies on GPS synchronization, including the financial market, the power grid, and the communications sector [20].

GPS time synchronization accuracy has been shown to be under 10 nanoseconds [5], and frequency accuracy is below $5 \mathrm{ppb}$ [6]. This is well within the most stringent frequency and time synchronization requirements defined in Table I. Moreover, the location accuracy of a GPS receiver tracking the GPS L1 C/A civilian signal is under 10 meters [11]. 
GPS is not without its drawbacks. Compared to terrestrial wireless communication systems, GPS transmits a comparatively weak signal. A typical GPS L1 C/A signal has a received signal strength around $-155 \mathrm{dBW}$ [11], which for a typical receiver with a GPS noise floor of $-204 \mathrm{dBW} / \mathrm{Hz}$, corresponds to $C / N_{0}=-155-(-204)=49 \mathrm{~dB}-\mathrm{Hz}$. Effective indoor GPS positioning requires the ability to acquire and track under severe $20-45 \mathrm{~dB}$ attenuation caused by building absorption [21]. Unaided, standalone GPS receivers can only acquire a GPS signal with $C / N_{0}$ down to about $29 \mathrm{~dB}-\mathrm{Hz}$, and thus can only withstand up to about $20 \mathrm{~dB}$ of attenuation. For this reason, standalone GPS is not a viable solution for femtocell synchronization and localization. However, by providing aiding information to a GPS receiver, the two techniques discussed below provide gains beyond this limit.

1) The A-GPS solution: Assisted GPS (A-GPS) uses a network-based approach to improve the acquisition performance of GPS receivers. Under A-GPS, the cellular network provides the receiver with the GPS databits, which allows it to coherently integrate a signal beyond the $20 \mathrm{~ms}$ data bit interval [16]. The network also provides the receiver with a coarse time and position estimate. All of this information allows the receiver to increase its search sensitivity as well as shrink its GPS time-frequency search space, shortening its time-to-firstfix (TTFF). State-of-the-art GPS chips employing A-GPS are claimed to be capable of acquiring signals with $C / N_{0}=14$ $\mathrm{dB}-\mathrm{Hz}$ [12]. This means that A-GPS provides about a $15 \mathrm{~dB}$ improvement in signal sensitivity over standalone GPS.

The limit of A-GPS comes at the point when the local clock in the receiver drifts significantly in phase (i.e., 1/4 cycle or more) during the coherent integration interval. Due to this phase drift, correlations with the incoming signal eventually become noncoherent, after which time further coherent integration degrades receiver sensitivity. An improvement in sensitivity can still be made but would require incoherent averaging of coherently integrated segments. This improvement, however, offers significantly lower gains in sensitivity than would be achievable with one longer coherent segment. Due to cost and size constraints, most femtocells employ low quality temperature-controlled crystal oscillators (TCXOs) as their local clock. These devices only remain coherent at GPS frequencies for about 400 milliseconds, limiting the feasible coherent integration time to this amount.

2) The EGPS Solution: Enhanced GPS (EGPS) uses the AGPS technique of receiving aiding information from the cellular network but more tightly couples timing and positioning measurements made from signals into the GPS receiver [16], [17]. EGPS can extract microsecond-level timing and sub-100 meter level positioning from the cellular signals. This finelevel aiding allows the GPS receiver to substantially shrink its time-frequency search space. However, this offers only mild improvements in sensitivity beyond A-GPS. The time aiding provided by EGPS has variations on the order of microseconds, far too much to allow the receiver to compensate for its local clock variations. This means that, like A-GPS, EGPS cannot extend the coherent integration interval beyond what the undisciplined local clock will allow.

\section{Tightly-Coupled Opportunistic NAVIGation}

Tightly-Coupled Opportunistic Navigation, or TCON, is a strategy for exploiting the frequency stability, transmit location, and timing information of non-GPS signals of opportunity to assist GPS-synchronized femtocells in weak-signal environments [15]. "Tightly-Coupled" means that the GPS signal and the signal of opportunity are downmixed with the same oscillator and sampled synchronously. "Opportunistic Navigation" means that the femtocell searches for opportune signals from which to extract navigation and timing information. TCON makes optimal use of GPS and other signals of opportunity. It does this by fusing observables from GPS and SOPs at the carrier-phase level in a centralized estimator (e.g., Kalman filter).

Two benefits of TCON most relevant to femtocells are (1) frequency stability transfer [22], which increases the coherence time of the femtocell's local clock, and (2) precise aiding of time and frequency information, which shrinks the GPS signal search space. These benefits "fall out" of a full TCON implementation where the centralized estimator takes in observations from SOPs and from GPS and drives all tracking loops. A full analysis of the TCON estimator's behavior cannot be presented here; however, the "fall out" benefits can be understood and, indeed, implemented, in isolation from the full TCON implementation. It is by exploiting these benefits that a femtocell could acquire and track GPS signals with $C / N_{0}$ as low as $5 \mathrm{~dB}-\mathrm{Hz}$, approximately a $9-\mathrm{dB}$ improvement over state-of-the-art A-GPS and EGPS solutions.

\section{A. TCON Benefit: Frequency Stability Transfer}

Frequency stability transfer provides a way to increase the coherence time of a femtocell's local oscillator [22]. Instead of replacing a femtocell's inexpensive TCXO with a more stable but expensive ovenized crystal oscillator (OCXO), one can configure the femtocell to create a "synthetic oscillator" by correcting phase errors during coherent integration based on a comparison with a non-GPS signal of opportunity whose carrier frequency has OCXO-level stability. In other words, the femtocell's TCXO "leans" on this signal of opportunity for increased frequency stability.

A model for frequency stability transfer fits naturally into a general GPS receiver framework as shown in Fig. 1. To extend the coherent summation duration, the rotation block applies a phase correction to each intermediate accumulation $S$ : each $S$ is rotated by an estimate $\Delta \phi$ of the local-oscillator-induced phase change over the intermediate accumulation interval. The phase correction term applied to $S_{j}$ at the $j$ th intermediate accumulation interval is

$$
\Delta \phi[j]=\left(\frac{f_{\mathrm{GPS}}}{f_{\mathrm{AID}}}\right) \cdot\left(\phi_{\mathrm{AID}}\left[n_{j}\right]-\phi_{\mathrm{AID}}\left[n_{j-1}\right]\right) .
$$

If the aiding signal is generated by a stable frequency source, and if its local phase measurement is referenced to the same local oscillator that drives the phase of $S$, then the aiding 


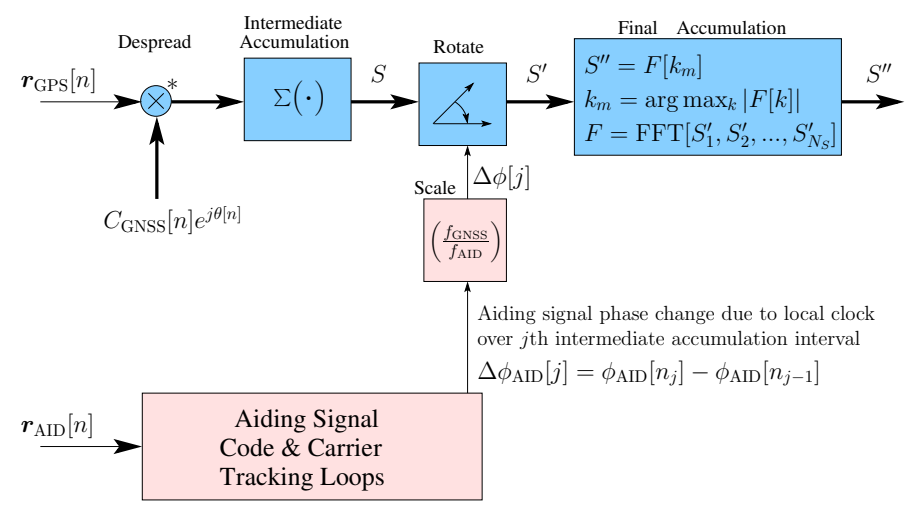

Fig. 1. A block diagram illustrating frequency stability transfer that makes use of two parallel acquisition and tracking paths, one for a GPS signal and one for an aiding signal. The aiding signal's phase corrections compensate for the local-oscillator-induced phase variations over each intermediate accumulation interval.

signal's phase shift $\Delta \phi_{\mathrm{AID}}$, after scaling by the ratio of the GPS and aiding frequencies $f_{\mathrm{GPS}} / f_{\mathrm{AID}}$, provides an estimate $\Delta \phi$ of the local oscillator's phase shift over each intermediate accumulation interval. Applying $\Delta \phi$ to $S$ to correct for this phase shift and repeating for each interval generates a sequence of phase-corrected accumulations $S_{1}^{\prime}, \ldots, S_{N_{S}}^{\prime}$.

The FFT-based final accumulation operation performs two tasks: (1) it removes any constant phase ramp that may be present in the sequence $S_{1}^{\prime}, \ldots, S_{N_{S}}^{\prime}$ due to a possible offset between the aiding signal's advertised and actual transmitted center frequency, and (2) it sums this sequence together, completing the coherent summation process and, ultimately, providing the femtocell with a coherent integration interval beyond what its unaided local clock would otherwise allow. This leads to increased GPS signal search sensitivity.

\section{B. TCON Benefit: Constraining the Search Space}

Absolute frequency and time information provided by the signals of opportunity can be coupled into the femtocell and used to shrink the GPS signal acquisition search space. The search space spans two dimensions: time (code-phase) and frequency. A receiver divides this space into a 2-D grid and searches each cell for the GPS signal. The larger the search space, the more cells that must be searched. TCON exploits the signals of opportunity to help make this search space as small as possible. In essence, the femtocell can use the accurate frequency of the SOP to narrow its frequency uncertainty and can use the timing information within the SOP to narrow its time (or range) uncertainty to the GPS satellites.

Even with a reduced search space, a femtocell must integrate in each search cell for a predetermined amount of time in order to confirm or deny the presence of a GPS signal with a desired probability of detection $P_{\mathrm{D}}$ and a desired probability of false alarm $P_{\mathrm{F}}$. Suppose that the indoor GPS signal has been attenuated to $C / N_{0}=5 \mathrm{~dB}-\mathrm{Hz}$ and that the femtocell is trying to coherently integrate to recover enough GPS signal power to acquire and track. A natural question to ask is the following: how long does the femtocell need to coherently integrate? Following the analysis outlined in [22], for a GPS signal search with a time uncertainty window of $6 \mu \mathrm{s}$ and frequency uncertainty window of $0.5 \mathrm{~Hz}$ (these values will be justified later in Section VI-E), a femtocell would need to be coherently integrate for approximately 7 seconds to reliably detect a GPS signal with $C / N_{0}=5 \mathrm{~dB}-\mathrm{Hz}$.

\section{SIGNALS OF OPPORTUNITY}

Not all signals of opportunity are suitable for TCON. This section considers three candidate signals of opportunity available to femtocells.

\section{A. Iridium Communication Satellite Signals}

The Iridium communication system is a constellation of 66 interlinked low earth orbit satellites designed to provide telephone services almost anywhere on earth [23]. Because they are at low-earth orbit, their $C / N_{0}$ is greater than that of GPS, often exceeding $60 \mathrm{~dB}-\mathrm{Hz}$. Iridium signals are modulated using a stable OCXO on-board the satellite, making them a good candidate for TCON.

The signals have a time-division multiple access (TDMA) and TDD modulation structure. This means that the carrier phase of the signal is available only during discrete bursts. For frequency stability transfer, a continuous-time carrier phase is required. This continuous carrier phase must be "stitched together" from the observed carrier phase and phase rate during each burst. As a rule of thumb for Iridium signals, continuous carrier recovery is possible if the phase drift between bursts does not exceed 1/8 of a cycle. The algorithms required to optimally stitch these bursts together are the subject of ongoing research by the authors and will be the topic of a future paper.

\section{B. High-Definition Television (HDTV) Signals}

High-definition television (HDTV) is another potential signal of opportunity for TCON. In fact, previous work in this area has shown digital television signals to be a very accurate augmentation to GPS for positioning [24], [25]. HDTV signals are exceptionally strong and are available in all major metropolitan areas in the U.S. The received signal strength of HDTV signals is about $-104 \mathrm{dBW}$ [25], a power advantage of about $50 \mathrm{~dB}$ over GPS. Also, the U.S. ATSC digital television standard mandates synchronized transmitters [24]. To comply with this requirement, the transmitter oscillator is relatively stable and imparts this stability onto the signals themselves.

\section{Cellular CDMA Signals}

Cellular code division multiple access (CDMA) signals are also strong candidate signals of opportunity for TCON because: (1) they are available in both urban and rural environments; (2) they are designed to penetrate buildings; (3) they have a dataless pilot channel that is referenced to a highly-stable clock; and (4) they embed GPS-referenced time of transmission within their synchronization channel.

Under the CDMA2000 standard, base stations are required to maintain time accuracy to within 10 microseconds of GPS time [26]. Many CDMA operators use highly-stable, GPSsynchronized oscillators in their CDMA base stations to meet 
this requirement. The base stations continually transmit a dataless pilot channel to synchronize with mobile devices. This pilot channel is derived directly from the base station's stable timebase, providing a receiver with a reliable, steady, and accurate frequency reference that can be acquired and tracked without the need for databit synchronization.

\section{CDMA-BASED TCON FOR FEMTOCELLS}

In the introduction, it was claimed that a 9 -dB gain over state-of-the-art GPS sensitivity capabilities is needed in order for a femtocell to acquire and track GPS signals in $90 \%$ of indoor residential environments. TCON was introduced as a strategy to achieve this gain and three viable signals of opportunity for TCON were presented. This section will establish that cellular CDMA, which has been selected as a vehicle for a TCON proof-of-concept, can be used within the TCON framework to achieve this $9-\mathrm{dB}$ gain in sensitivity for femtocells.

\section{A. Experimental Setup}

In a dedicated CDMA data collection and analysis campaign at The University of Texas at Austin, cellular CDMA signals were recorded and analyzed. A National Instruments vector signal analyzer, referenced to a highly-stable GPS-disciplined oscillator in the laboratory, was used to record signals from three CDMA base stations operated by Verizon Wireless, Sprint, and Cricket Wireless. The software-defined GRID receiver [27] was then used to track each received signal. The signals were analyzed for their frequency stability, their frequency accuracy, and their time accuracy. The frequency stability analysis determines the limit of the coherent integration time achievable through employing CDMA signals as aiding signals in frequency stability transfer. The frequency and timing accuracy governs the extent to which a TCONaided femtocell can shrink its GPS Doppler frequency and code-phase search space.

\section{B. Frequency Stability of a CDMA signal}

First, a frequency stability analysis was performed on the CDMA signals. The GRID receiver tracked and provided the beat carrier phase time history $\phi(t)$ for each signal. A useful metric for comparing oscillator stability is the coherence function, $C_{\mathrm{coh}}$ :

$$
C_{\mathrm{coh}}(T)=\left|\frac{1}{T} \int_{0}^{T} e^{j \phi(t)} d t\right|, \quad 0 \leq C_{\mathrm{coh}}(T) \leq 1 .
$$

The coherence function expresses the effects of variations in $\phi(t)$ during a coherent integration period $T$. When compared against an ideal clock, the coherence time $T_{\text {coh }}$ of a signal is defined as the time $T$ at which the mean of the square of the coherence function $\left\langle C_{\mathrm{coh}}^{2}(T)\right\rangle$ drops below 0.5. Signals of opportunity cannot be used within frequency stability transfer to support coherent integration durations beyond their $T_{\text {coh }}$. $\left\langle C_{\mathrm{coh}}^{2}(T)\right\rangle$ was computed on all three signals as a function of $T$. The results are shown in Fig. 2. Also shown is the meansquared coherence of a typical TCXO and OCXO. The plot

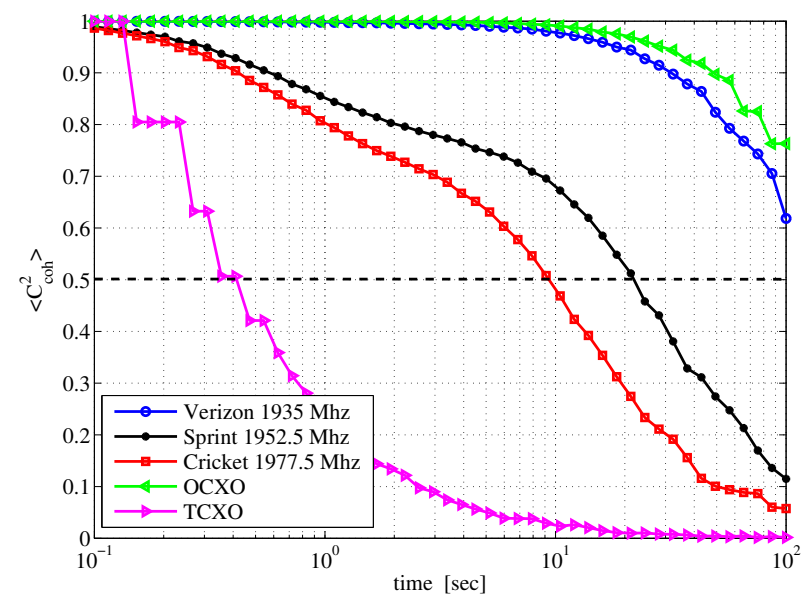

Fig. 2. Mean-squared coherence vs. time for a TCXO, an OCXO, and three CDMA signals.

TABLE II

Mean and Standard Deviation of CDMA Frequency ERror

\begin{tabular}{|c|c|c|c|}
\hline Carrier & Freq. $[\mathrm{Mhz}]$ & Mean $[\mathrm{Hz}]$ & Std. Dev. $[\mathrm{Hz}]$ \\
\hline Verizon & 1935 & 0.006 & 0.012 \\
\hline Sprint & 1952.5 & -0.001 & 0.038 \\
\hline Cricket & 1977.5 & 0.100 & 0.058 \\
\hline
\end{tabular}

reveals that all three CDMA signals as well as the OCXO remain coherent as long as the required coherent integration duration is not above 9 seconds. The TCXO however, only remains coherent up to 400 milliseconds.

\section{Frequency Accuracy of a CDMA signal}

Next, the frequency accuracy of the three CDMA signals was characterized. At each sample time, the GRID receiver calculated the instantaneous carrier frequency offset of each signal from its nominal center frequency. These instantaneous measurements were averaged with a 7 second sliding window to filter out front-end thermal noise. The mean and standard deviation of the averages over the 40 minute recordings are given in Table II. All three CDMA signals maintain their frequency accuracy to within $0.3 \mathrm{~Hz}$ of their nominal center frequency (computed by mean $+/-3$ standard deviations). This information would allow a TCON-enabled femtocell tracking CDMA signals to shrink its Doppler frequency search range such that it only searches within about $0.3 \mathrm{~Hz}$ of the GPS nominal frequency for the presence of a GPS signal.

\section{Timing accuracy of a CDMA signal}

Last, the timing accuracy of the three CDMA signals was characterized. This is a measure of how closely the base stations maintain their individual system time to GPS time. By differencing the time of transmission embedded in each signal from the GPS-timestamped time of reception, the timeof-flight (TOF) plus any deviation in the base station's system time from GPS time was calculated. Table III shows the results of this time difference for the three signals. The results show that the Cricket and Verizon base stations maintain their 
TABLE III

MEAN AND STANDARD DEVIATION CDMA TOF + TIMING ERRor

\begin{tabular}{|c|c|c|c|}
\hline Carrier & Freq. $[\mathrm{Mhz}]$ & Mean $[\mu s]$ & Std. Dev. $[\mu s]$ \\
\hline Cricket & 1977.5 & 3.773 & 0.031 \\
\hline Verizon & 1935 & 2.968 & 0.016 \\
\hline Sprint & 1952.5 & 40.745 & 0.035 \\
\hline
\end{tabular}

system time to within $4 \mu s$ of GPS time. Their true time accuracy is even better than this since the TOF could be factored out, if known. For example, if a femtocell is able to obtain a coarse approximation of its range to the base station, it can estimate the TOF and add this amount to the time of transmission extracted from the signal to obtain a more accurate sense of time. Nonetheless, even a $4 \mu s$ error will substantially decrease the code-phase search space of a femtocell. The Sprint base station TOF is shown to be over 40 $\mu s$. It is assumed that this base station maintained a constant offset from GPS time. Such an offset, if known, could be provided to the femtocell or characterized on-the-fly.

\section{E. Projected Sensitivity Gains of CDMA-based TCON}

In Section IV-B it was calculated that a coherent integration time of approximately 7 seconds would be needed for a femtocell to acquire a GPS signal with $C / N_{0}=5 \mathrm{~dB}-\mathrm{Hz}$. Three assumptions were made in this calculation. The first was that the femtocell could provide a frequency that is accurate to within $0.5 \mathrm{~Hz}$ of the nominal GPS frequency. The second was that it could provide time accurate to within $6 \mu s$ of true GPS time. The third was that the TCON-aided femtocell oscillator remained largely coherent over the 7 second period of coherent integration. The CDMA signal stability and accuracy results suggest that the three signals tested will enable a TCONaided femtocell to meet these assumptions. If these signals are representative of CDMA signals nationwide, then cellular CDMA looks very promising as a signal of opportunity and, consequently, can be exploited within the TCON framework to enable a femtocell to acquire and track GPS signals with $C / N_{0}$ as low as $5 \mathrm{~dB}-\mathrm{Hz}$.

\section{CONCLUSION}

A preliminary study of the Tightly-Coupled Opportunistic Navigation strategy indicates that a TCON-aided femtocell could acquire and track GPS signals down to $5 \mathrm{~dB}-\mathrm{Hz}$. By harnessing the frequency and time information of CDMA signals, a femtocell operating within the TCON framework can achieve the necessary gain in sensitivity to support GPS acquisition and tracking in $90 \%$ of residences. This allows the unparalleled time and positioning accuracy provided by GPS to be extended to a vast majority of residential femtocell deployments, enabling TCON-aided femtocells to meet all time, frequency, and location requirements set forth by cellular standards and regulatory agencies.

\section{REFERENCES}

[1] R. Kim, J. Kwak, and K. Etemad, "Wimax femtocell: requirements, challenges, and solutions," Communications Magazine, IEEE, vol. 47, no. 9, pp. 84-91, 2009.
[2] Rakon Corporation, "Rakon femtocell timing solutions," 2008, http://www.rakon.com/resources/Documents/Rakon\%20Femtocell\%20 Fact\%20Sheet.pdf.

[3] J. Edwards, "Implementation of network listen modem for WCDMA femtocell," in Cognitive Radio and Software Defined Radios: Technologies and Techniques, 2008 IET Seminar on. IET, pp. 1-4.

[4] J. Eidson and K. Lee, "Ieee 1588 standard for a precision clock synchronization protocol for networked measurement and control systems," in Sensors for Industry Conference, 2002. 2nd ISA/IEEE. IEEE, 2002, pp. $98-105$.

[5] W. Lewandowski, J. Azoubib, and W. Klepczynski, "GPS: Primary tool for time transfer," Proceedings of the IEEE, vol. 87, no. 1, pp. 163-172, 1999.

[6] S. Smith and J. Moore, "A precision, low-cost GPS-Based transmitter synchronization scheme for improved AM reception," Broadcasting, IEEE Transactions on, vol. 55, no. 1, pp. 71-78, 2009.

[7] F.C.C. Docket No., "94-102," Revision of the commission's rules to ensure compatibility with enhanced 911.

[8] — , "07-114," In the Matter of Wireless E911 Location Accuracy Requirements.

[9] J. Kim, J. Lee, and C. Park, "A mitigation of line-of-sight by TDOA error modeling in wireless communication system." in International Conference on Control, Automation and Systems, 2008. IEEE.

[10] "Femtocell synchronization and location, a Femto Forum topic brief," Femto Forum Whitepapers, vol. 1, no. 15, June 2010, http://www.femtoforum.org/femto/pdfs01.php.

[11] P. Misra and P. Enge, Global Positioning System: Signals, Measurements, and Performance. Lincoln, Massachusetts: Ganga-Jumana Press, 2006.

[12] "SiRFstarIV GSD4t datasheet," CSR, Cambridge, UK.

[13] Rosum Corporation, "In-building location, timing, and frequency coverage analysis of A-GPS and TV-GPS for femtocell applications," Whitepaper, 2009.

[14] Mitelman, A., et al., "The Nordnav indoor GNSS reference receiver," in Proceedings of the ION GNSS Meeting, 2001, pp. 2494-2502.

[15] K. Pesyna, Z. Kassas, J. Bhatti, and T. E. Humphreys, "Tightly-coupled opportunistic navigation for deep urban and indoor positioning (to appear)," in Proceedings of the ION GNSS 2011, 2011.

[16] R. Rowe, P. Duffett-Smith, M. Jarvis, and N. Graube, "Enhanced GPS: The tight integration of received cellular timing signals and GNSS receivers for ubiquitous positioning," in Position, Location and Navigation Symposium. IEEE/ION, 2008, pp. 838-845.

[17] P. J. Duffett-Smith and B. Tarlow, "E-GPS: Indoor mobile phone positioning on GSM and W-CDMA," in Proceedings of the ION GNSS Meeting, 2005.

[18] V. Chandrasekhar, J. Andrews, and A. Gatherer, "Femtocell networks: a survey," Communications Magazine, IEEE, vol. 46, no. 9, pp. 59-67, 2008.

[19] Freedman, M.J., et al., "Geographic locality of IP prefixes," in Proceedings of the 5th ACM SIGCOMM conference on Internet Measurement. USENIX Association, 2005, pp. 13-13.

[20] National Coordination Office for Space-Based Positioning, Navigation, and Timing, "GPS timing applications," http://www.gps.gov/applications/timing/.

[21] G. Dedes and A. Dempster, "Indoor GPS positioning-challenges and opportunities," in Vehicular Technology Conference, 2005. VTC-2005Fall. 2005 IEEE 62nd, vol. 1. IEEE, 2005, pp. 412-415.

[22] K. Wesson, K. Pesyna, J. Bhatti, and T. E. Humphreys, "Opportunistic frequency stability transfer for extending the coherence time of GNSS receiver clocks," in Proceedings of the ION GNSS Meeting. Portland, Oregon: Institute of Navigation, 2010.

[23] K. Maine, C. Devieux, and P. Swan, "Overview of iridium satellite network," in WESCON/'95 Conference. IEEE, nov 1995, p. 483.

[24] M. Rabinowitz and J. Spilker Jr, "A new positioning system using television synchronization signals," Broadcasting, IEEE Transactions on, vol. 51, no. 1, pp. 51-61, 2005.

[25] J. Do, M. Rabinowitz, and P. Enge, "Performance of hybrid positioning system combining GPS and television signals," in Position, Location, And Navigation Symposium, 2006 IEEE/ION, 2006, pp. 556-564.

[26] Recommended Minimum Performance Standards for cdma2000 Spread Spectrum Base Stations, C.S0010-B, 3rd Generation Partnership Project 2 "3GPP2" Std., February 2004.

[27] T. E. Humphreys, J. Bhatti, T. Pany, B. Ledvina, and B. O'Hanlon, "Exploiting multicore technology in software-defined GNSS receivers," in Proceedings of the ION GNSS Meeting, 2009. 